\title{
Influence of Thermal Radiation on a Transient MHD Couette Flow through a Porous Medium
}

\author{
I. G. Baoku ${ }^{1 \dagger}$, C. Israel-Cookey ${ }^{2}$ and B. I. Olajuwon ${ }^{3}$ \\ ${ }^{1}$ Department of Mathematical Sciences, Crescent University, Abeokuta, Nigeria. \\ ${ }^{2}$ Department of Mathematics \& Computer Science, Rivers State University of Science and Technology, \\ Port Harcourt, Nigeria. \\ ${ }^{3}$ Department of Mathematics, University of Agriculture, Abeokuta, Nigeria. \\ †Corresponding Author Email: ishola1@gmail.com
}

(Received February 8, 2010; Accepted November 4, 2010)

\begin{abstract}
The paper examines transient MHD Couette flow of an electrically conducting fluid in the presence of an applied transverse magnetic field and thermal radiation through a porous medium. The dimensionless governing equations of the flow are coupled non-linear partial differential equations and are solved by an efficient and unconditionally stable finite difference scheme of Crank-Nicolson type. The influence of the medium permeability is also assessed. The velocity and temperature profiles for the flow are studied for various interesting parameters of Prandtl number, Nahme number and Hartmann number, and are presented graphically. The results show that the thermal radiation has appreciable influence on the flow.
\end{abstract}

Keywords: MHD Couette flow, Thermal radiation, Porous medium, Prandtl number, Nahme number and Hartmann number.

\section{NOMENCLATURE}

$\begin{array}{ll}b & \text { thickness of the fluid layer } \\ B_{0} & \text { magnetic induction } \\ \mathrm{C}_{\mathrm{p}} & \text { specific heat at constant pressure } \\ \mathrm{fr}_{\mathrm{r}} & \text { fluid resistance } \\ \mathrm{M} & \text { Hartmann number } \\ \mathrm{Na} & \text { Nahme number } \\ \mathrm{Pr} & \text { Prandtl number } \\ q^{\prime} & \text { radiactive heat flux } \\ \mathrm{r} & \text { convergent term } \\ \mathrm{r}_{1} & \text { radius of the inner cylinder } \\ \mathrm{r}_{2} & \text { radius of the outer cylinder } \\ \mathrm{R} & \text { radiation parameter } \\ \mathrm{t} & \text { dimensionless time } \\ \mathrm{t}^{*} & \text { local time } \\ \mathrm{T} & \text { local temperature } \\ \mathrm{T}_{0} & \text { reference temperature } \\ \mathrm{T} & \text { temperature at time } \mathrm{t} \\ \Delta \mathrm{t} & \text { time step }\end{array}$

\section{INTRODUCTION}

In recent years, considerable progress has been made in the study of the thermophysical properties affecting Magnetohydrodynamic (MHD) flow due to its applications in many scientific and engineering studies. Under certain conditions, a moving electrically conducting fluid itself induces a magnetic field so that an MHD phenomenon may not require the presence of

$\begin{array}{ll}\Delta \mathrm{t} & \text { time step } \\ \mathrm{u} & \text { dimensionless velocity } \\ \mathrm{U} & \text { linear velocity of the outer cylinder } \\ \mathrm{u}^{*} & \text { local fluid velocity } \\ \mathrm{v} & \text { velocity of flow direction } \\ \mathrm{y} & \text { flow direction } \\ \mathrm{y}^{*} & \text { local flow direction } \\ \alpha^{2} & \text { absorption coefficient } \\ \theta & \text { dimensionless temperature } \\ \kappa^{*} & \text { thermal conductivity } \\ \lambda^{*} & \text { permeability of the porous medium } \\ \lambda^{\prime} & \text { dimensionless permeability } \\ \mu & \text { dynamic viscosity } \\ \mu_{0} & \text { dynamic viscosity at To } \\ \nu & \text { kinematic viscosity } \\ \rho & \text { fluid density } \\ \sigma_{0} & \text { magnetic permeability } \\ \omega & \text { angular velocity }\end{array}$

an external magnetic field. However, this internallygenerated magnetic field may be very small to simplify the problem. Also, as magnetic field has its source by either moving charges or changing electric field, the magnetic field of the earth undergoes complex changes due to electric currents flowing in the ionosphere. The study of MHD flow and magnetic field has gained the attention of many researchers because of their applications in the design of heat exchangers, MHD 
pumps, induction pumps, MHD generators, nuclear reactors, in oil exploration and in space vehicle propulsion.

Chamka (2003) examined a generalized problem of heat and magnetic fields on MHD flow. He investigated analytical solutions for the problem of heat and mass transfer with steady flow of an electrically conducting and heat generating / absorbing fluid on a uniform moving vertical permeable surface in the presence of a magnetic field. He concluded that the fluid velocity decreases as the Prandtl number, Schmidt number or strength of the magnetic field is increased. IsraelCookey and Sigalo (2003) reported the study of an unsteady MHD free-convection and mass transfer flow past an infinite heated porous vertical plate with timedependent suction in an optically thin environment.

Also, Gbadeyan and Dada (1998) considered the effect of variable fluid properties and radiative MHD flow of a fluid in a vertical channel. Alagoa et al. (1999) investigated the problem of MHD free-convection flow with radiation heat transfer in a porous medium. It was shown that the field is affected mainly by radiation and convection parameters in addition to magnetic factors. Hazeem (2006) examined the effect of variable viscosity on the transient MHD Couette flow of dusty fluid with heat transfer between parallel plates. He showed some important effects for the variable viscosity and the uniform magnetic field on the transient flow and heat transfer of both the fluid and dust particles.

Similarly, heat transfer by radiation occurs between solid surfaces, although radiation from gases is also possible. Solid radiates over a wide range of wavelengths while some gases emit and absorb radiation on certain wavelengths only. Hence, thermal radiation has effects on electrically conducting fluid at high operating temperature. When radiative heat transfer sets in, a high temperature fluid will generate heat by thermal radiation and the radiation can actually affect the motion and more importantly the energy mechanism of the fluid. Raptis and Massalas (1998) studied the radiation effect on the unsteady MHD flow of an electrically conducting viscous fluid past a plate. Chamka (2000) investigated thermal radiation and buoyancy effects on hydromagnetic flow over an accelerating permeable surface with heat source or sink. Takhar et al. (2001) analyzed the unsteady laminar MHD flow and heat transfer in the stagnation region of an impulsively spinning and translating sphere in the presence of buoyancy forces. They found out that the surface shear stresses in the longitudinal and rotation directions and heat transfer increase with time, magnetic field, buoyancy parameter and rotation parameter.

In the same vein, Makinde and Mhone (2005) investigated the combined effect of a transverse magnetic field and radiactive heat transfer to unsteady flow of a conducting optically thin fluid through a channel filled with saturated porous medium and nonuniform walls temperature. The velocity and temperature profiles are obtained analytically and used to compute the wall shear stress and rate of heat transfer at the channel walls. Anjali Devi and Ganga (2010) studied effects of viscous and Joule dissipation on MHD nonlinear flow and heat transfer past a stretching porous surface embedded in a porous medium under a transverse magnetic field. Analytical results of the transformed MHD boundary layer equations were obtained and significant effects of dissipation and porosity were enunciated.

More so, flows in porous media have gained the attention of many researchers because of their applications in geothermal, oil reservoir engineering and astrophysics. Sharma and Mathur (1995) investigated steady laminar free-convection flow of an electrically conducting fluid along a porous shot vertical infinite plate in the presence of source or sink. Also, Sharma and Singh (2009) analyzed effects of variable thermal conductivity, heat source/sink on flow of a viscous incompressible electrically conducting fluid in the presence of uniform transverse magnetic field and variable free stream near a stagnation point on a non-conducting stretching sheet. They discussed the influence of the flow parameters used and their physical implications. Daskalaskis (1990) studied Couette flow through a porous medium of a high Prandtl number fluid with temperature dependent viscosity. He concluded that, in the steady state, the medium permeability $(\lambda)$ for both velocity and temperature profiles are positively skew and their skewness increases with $\lambda$. He also affirmed that the lower the permeability of the medium the faster the velocity but it does not significantly influence the temperature profile development. The influence of plate porosity on steady MHD flow profile is evident in the work of Makinde and Osalusi (2006) and Osalusi and Sibanda (2006). Min Chan et al. (2004) considered theoretically an initially quiescent, fluid saturated horizontal porous layer heated from below with constant heat flux. They used Darig's law as a model to the fluid motion and linear stability theory, and predicted the onset of buoyancy-driven convective flow.

The model under investigation considers the influence of thermal radiation on a transient MHD Couette flow through a porous medium in the presence of an applied uniform magnetic field and has not received any attention in literature. Hence, the present work studies the transient MHD Couette flow of a high Prandtl number fluid with temperature-dependent viscosity through a porous medium. The governing flow equations are solved by an implicit finite difference scheme of Crank-Nicolson type with $\mathrm{Pr}, \mathrm{Na}, \mathrm{M}$ and R as controlling parameters.

\section{Formulation of the Problem}

The geometry for the flow consists of two concentric infinite cylindrical surfaces with the outer surface having radius $\mathbf{r}_{2}$. The MHD fluid under investigation occupies the annular space of width $\mathbf{b}$ between the cylinders. The system is at rest at first instant and the two surfaces are at constant temperature $t_{0}$. At time $t^{*}=0$, the outer cylinder is suddenly set in motion and rotates with constant angular velocity $\omega$ while the inner cylinder of radius $\mathbf{r}_{1}$ is stationary in the presence of an applied external transverse magnetic field. 
The MHD fluid is considered to have a negligible internally-generated magnetic field and in the course of time, the fluid gradually participates in the motion and viscous dissipation increases the temperature. The electrically conducting fluid is presumed to have a very small electric current within the MHD fluid flow and the temperature of the two cylinders is maintained at the constant temperature $T_{o}$ by cooling. The thickness $\mathbf{b}$ of the fluid layer is assumed to be small compared with the outer cylinder so that the problem is identical to that of Couette flow with a negligible pressure gradient. The permeability is also assumed to be constant.

We reduce the complicated motion of a viscous fluid in a porous solid to that of the motion of a homogeneous fluid with some additional resistance $\mathbf{f}_{\mathbf{r}}$ and the nonhomogeneous medium is homogeneous with dynamical properties equal to the local averages of the original fluid continuum. This resistance is modelled as: $f_{r}=-\left(\frac{v}{\lambda^{*}}\right) u^{*}$ and the temperature law for the viscosity is taken as: $\mu=\mu_{0} \exp \left\{-\beta\left(T-T_{0}\right)\right\}$ where $\beta$ is a constant. The magnetic field parameter for the MHD flow is also represented by: $M=B_{0} \sqrt{\frac{\sigma_{0}}{\rho \nu}}$ and following Takhar et al. (1996), the radiative heat flux is: $\frac{\partial q}{\partial y^{*}}=4 \alpha^{2}\left(T^{\prime}-T_{0}\right)$. It is assumed that the flow we studied takes place in a porous medium and concerns a high viscosity fluid exhibiting a strong dependence on temperature and constant thermal conductivity. Hall effect, Joule heating and other variable thermo-physical parameters are assumed to be constants.

Under the above assumptions, the incompressible MHD fluid flow, relevant for the problem, is governed by the following equations:

$$
\begin{aligned}
& \frac{\partial v}{\partial y^{*}}=0 \\
& \frac{\partial u^{*}}{\partial t^{\circ}}=\frac{1}{\rho} \frac{\partial}{\partial y^{*}}\left(\mu \frac{\partial u^{*}}{\partial y^{*}}\right)-\frac{v}{\lambda^{*}} u^{*}+\frac{\sigma_{0} B_{0}^{2}}{\rho v} u^{*} \\
& \frac{\partial T}{\partial t^{*}}=\frac{K^{*}}{\rho C_{p}} \frac{\partial^{2} T}{\partial y^{* 2}}+\left(\frac{\mu}{\rho C_{p}}\right)\left(\frac{\partial u^{*}}{\partial y^{*}}\right)^{2}-4 \alpha^{2}\left(T^{\prime}-T_{\infty}\right)
\end{aligned}
$$

Subject to the initial and boundary conditions where Eq. (1) gives $\mathrm{v}(\mathrm{y})=\mathrm{v}_{0}$ :

$$
\begin{array}{ll}
t *<0, & u^{*}=0, \quad T=T \\
y^{*}=0, & u^{*}=0, \quad T=T 。 \\
y^{*}=b, & u^{*}=U, \quad T=T_{\circ}
\end{array}
$$

where $U$ is the linear velocity of the outer cylinder $\left(U=r_{2} \omega\right)$.

Introducing the following dimensionless parameters:

$$
\begin{aligned}
& y=\frac{y *}{b}, \quad \theta=\beta\left(T=T_{\mathrm{o}}\right), \quad t=\frac{k^{*} t^{*}}{\rho C_{p} b^{2}}, \quad U=\frac{u^{*}}{u}, \\
& \lambda=\frac{\lambda^{*}}{b^{2}}, \quad \operatorname{Pr}=\frac{\mu_{0} c_{p}}{\kappa^{*}}, \quad N a=\frac{\mu_{0} \beta U^{2}}{\kappa^{*}},
\end{aligned}
$$

we then transform the above governing equations into their dimensionless form:

$$
\begin{aligned}
& \frac{\partial u}{\partial t}=\operatorname{Pr}(1-\theta) \frac{\partial^{2} u}{\partial y^{2}}-\operatorname{Pr}\left(\frac{\partial \theta}{\partial y}\right)\left(\frac{\partial u}{\partial y}\right)-\operatorname{Pr}\left[\lambda^{\prime}(1-\theta)-M^{2}\right] u \\
& \frac{\partial \theta}{\partial t}=\frac{\partial^{2} \theta}{\partial y^{2}}+N a(1-\theta)\left(\frac{\partial u}{\partial y}\right)^{2}
\end{aligned}
$$

where $M=b B_{0} \sqrt{\frac{\sigma_{0}}{\mu_{0}}}$ and $R=2 \alpha b \sqrt{\frac{1}{\kappa^{*}}}$

The appropriate initial and boundary conditions are written as:

$$
\begin{aligned}
& t \leq 0, \quad y=0, \quad u=0, \quad \theta=0 ; \\
& t>0, \quad y=0, \quad u=0, \quad \theta=0 \text {; } \\
& y=1, \quad u=1, \quad \theta=0 .
\end{aligned}
$$

\section{Method OF Solution}

The transient non-linear coupled partial differential Eqs. (5) and (6) with the initial and boundary conditions (7) are solved by employing the finite difference scheme of Crank-Nicolson type which is discussed in Ganesan and Ekambayanan (1992), Ganesan and Palani (2002) and Kreyzig (2003). We discretize the governing Eqs. (5) and (6) based on the transient state conditions. The numerical method of finite difference scheme of CrankNicolson type does not restrict the value of $r$ to be chosen. The finite difference equations corresponding to these equations are given by:

$$
\begin{aligned}
& \left(2+2 r \operatorname{Pr}-2 r \operatorname{Pr} \theta_{i, j}\right) u_{i, j+1}-r \operatorname{Pr}\left(1-\theta_{i, j}\right) u_{i+1, j+1} \\
& -r \operatorname{Pr}\left(1-\theta_{i, j}\right) u_{i-1, j+1}= \\
& \left(2-2 r \operatorname{Pr}+2 r \operatorname{Pr} \theta_{i+1, j}-2 \operatorname{Pr} \Delta t \lambda^{\prime}+2 \operatorname{Pr} \Delta t \lambda^{\prime} \theta_{i, j}(8)\right. \\
& \left.+2 \operatorname{Pr} \Delta t M^{2}\right) u_{i, j}+r \operatorname{Pr}\left(1+\theta_{i, j}-2 \theta_{i+1, j}\right) u_{i+1, j} \\
& (2+2 r) \theta_{i, j+1}-r \theta_{i+1, j+1}-r \theta_{i-1, j+1}= \\
& {\left[2-2 r-2 r N a\left(u_{i+1, j}-u_{i, j}\right)^{2}-2 \Delta t R^{2}\right] \theta_{i, j}} \\
& +r \theta_{i+1, j}+r \theta_{i-1, j}+2 r N a\left(u_{i+1, j}-u_{i, j}\right)^{2}
\end{aligned}
$$

where $\mathrm{i}$ designates the grip point along the y-direction and $\mathrm{j}$ along the $\mathrm{t}$-direction and $\mathrm{r}=\Delta \mathrm{t} / \mathrm{h}^{2}$. Hence, the momentum and energy equations in finite difference equations are reduced to algebraic system of linear equations. The mesh size $\mathrm{h}$ is 0.2 with time step $\Delta \mathrm{t}=$ $0.33,0.001,0.01,0.05,0.0025$ and 0.5 . The values of $\mathrm{u}(\mathrm{y}, \mathrm{t})$ are known at all grid points when $\mathrm{t}=0$ from the initial conditions. Computations are carried out by moving along y-direction. After computing values corresponding to each $i$ at a time level, the values at the next time level are determined in similar manner.

These are the necessary and sufficient conditions for consistency and stability of the solutions. The implicit nature of Crank-Nicolson method is unconditionally stable and has local truncation error $\mathrm{O}\left[(\Delta \mathrm{t})^{2}+\mathrm{h}^{2}\right]$ which tends to zero as $\Delta \mathrm{t}$ and $\mathrm{h}^{2}$ tend to zero, and there is no drawback of conditionally stability from one level to the next. The implicit method gives stable solutions and requires matrix inversions which we did at step forward in time because this problem is an initial boundary value problem with a finite number of spatial grid points. Though, the corresponding difference 
equations do not automatically guarantee the convergence of the mesh $h \rightarrow 0$. To achieve maximum numerical efficiency, we used the tridiagonal procedure to solve the two point conditions governing the main coupled governing equations of momentum and energy. The convergence (consistency) of the process was quite satisfactory and the numerical stability of the method was guaranteed by the implicit nature of the numerical scheme. Hence, the scheme is consistent. Stability and consistency ensure convergence.

\section{Discussion OF THE RESUlts}

A MHD fluid flow problem for momentum and energy equations in a porous medium with thermal radiation in the presence of a transverse magnetic field is investigated in this work. Porous medium, viscous dissipation, magnetic field and thermal radiation effects are taken into consideration. The MHD governing equations of the problem are solved numerically and solutions are obtained for the dimensionless velocity and temperature profiles for prescribed controlling parameters. For the purpose of discussing the effects of various parameters on the flow profiles and the temperature distributions with the MHD fluid, numerical calculations have been carried out for different values of $\mathrm{M}, \mathrm{R}, \mathrm{Pr}$ and $\mathrm{Na}$, and results have been presented in graphical forms. The permeability parameters $\lambda^{\prime}=0$ corresponds to free flow; $\lambda^{\prime}=2$ corresponds to moderate permeability; $\lambda^{\prime} \geq 10$ denotes low permeabilities and $\lambda^{\prime}=\leq 1$ represents high permeabilities. The values of $\mathrm{M}$ were taken to be: 3 while $\lambda^{\prime}=2$ for $\mathrm{t}=0.01 ; 1$ while $\lambda^{\prime}=1$ for $\mathrm{t}=0.001 ; 5$ while $\lambda^{\prime}=5$ for $\mathrm{t}=0.5 ; 1$ while $\lambda^{\prime}=1$ for $\mathrm{t}=0.001 ; 5$ while $\lambda^{\prime}=5$ for $t=0.0001$ in the case of $\operatorname{Pr}=1000$. However, when $\operatorname{Pr}=100$, the values of $\mathrm{M}$ were taken to be: 1 while $\lambda^{\prime}=10$ for $\mathrm{t}=0.001 ; 3$ while $\lambda^{\prime}=2$ for $\mathrm{t}=$ $0.001 ; 3$ while $\lambda^{\prime}=2$ for $t=0.01$ and 5 while $\lambda^{\prime}=5$ for $\mathrm{t}=0.001$

Furthermore, the transient velocity profiles are significantly governed by the Prandtl number and permeability parameter with the magnetic field parameter. The transient velocity profiles are significantly governed by the Prandtl number and permeability parameter with reference to the Hartmann number. The effects of Hartmann number on the velocity profiles are shown in Figs. 1-4. Influence of the high temperature is noticeable in the velocity profiles because of the fact that the conducting fluid viscosity is temperature-dependent and viscous dissipation increases with temperature increase. Also, the temperature distributions depict energy mechanism of the MHD flow with thermal radiation and Nahme number effects as displayed Figs. 5-6.

Figure 1 shows the effect of permeability parameter and Hartmann number on the dimensionless velocity profile. It is found that corresponding increase in the permeability parameter leads to a corresponding increase in the velocity profile with $\operatorname{Pr}=100$. This is because the porosity of the medium allows more fluid flow at $\mathrm{t}=0.001$. However, the result is different when there is absence of magnetic field parameter with free flow as the velocity profile increases tremendously when $\mathrm{M}=0$ than when there is influence of magnetic field on the flow. This is because the effect of magnetic field parameter on the velocity profile is to reduce the transverse velocity of the flow. The imposition of the magnetic field normal to the flow direction is responsible. This magnetic field gives rise to a resistive force and slows down the movement of the fluid. Hence, the lower velocity profiles for the cases of $\mathrm{M}=1$ when $\lambda^{\prime}=1$ and 10 at the time step of 0.001 . All these are noticeable when the transverse magnetic field is uniformly applied for the MHD flow. As the magnetic flux becomes higher, there is corresponding decrease in the velocity profiles.

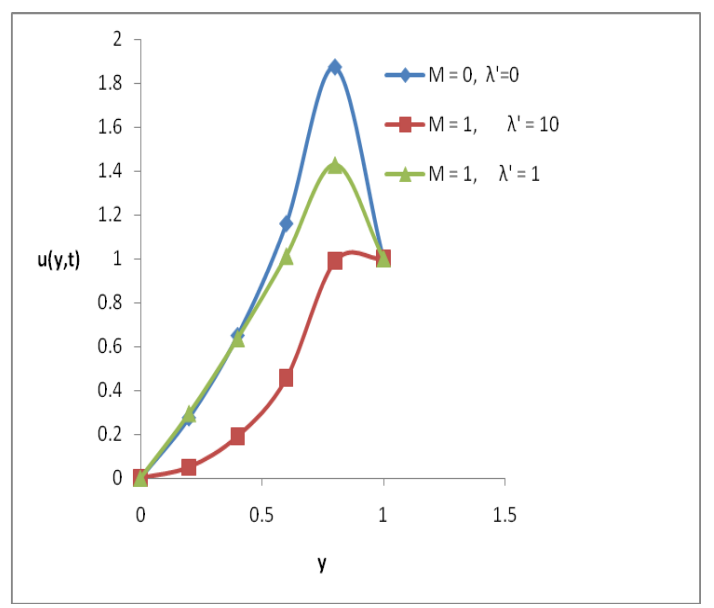

Fig. 1. Velocity profiles against y at $\operatorname{Pr}=100$.

Figure 2 analyzes the effect of Hartmann on the velocity profile when the medium permeability is moderate. It shows that increase in Hartmann number give rise to corresponding decrease in the velocity profiles of the fluid flow at $\operatorname{Pr}=100$ in the transient state. The Hartmann number represents the importance of magnetic field on the flow.

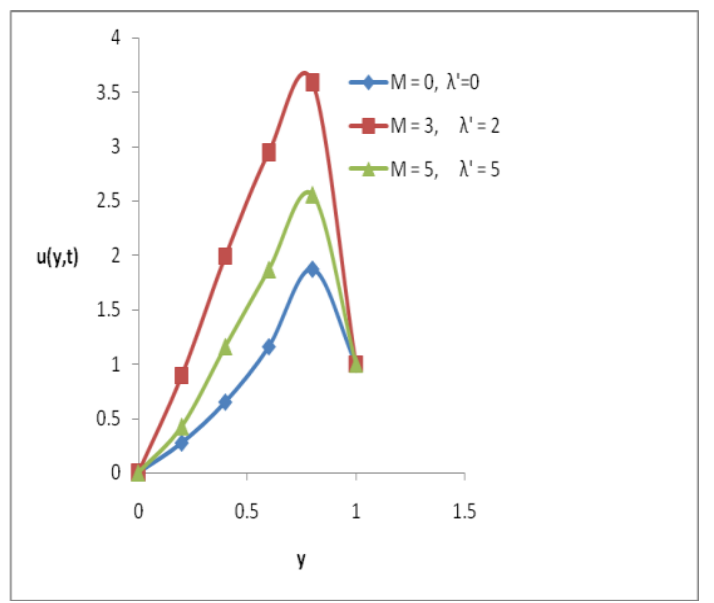

Fig. 2. Dimensionless velocity profiles against $y$ at $\operatorname{Pr}=100$ with different $M$ and $\lambda^{\prime}$

The presence of transverse magnetic field sets in Lorentz force which results in retarding force on the velocity field and therefore as Hartmann number increases, so does the retarding force on the velocity field and hence the velocity profiles decrease. The increase in magnetic field parameter which signifies 
corresponding increase of Lorentz force in which more lines of force are frozen into conducting fluid and are carried along with it, thereby reducing the velocity of the fluid flow as the Hartmann number increases. Also, the reliability of the results was also tested by comparing the results obtained in the special case of $\lambda$, $=0$ (free flow) against those of Eckert and Faghri (1986), who carried out a similar calculation for the simpler case of a homogeneous flow without porous medium and magnetic field (i.e. $\lambda^{\prime}=0$ and $M=0$ ) when the Prandtl number is 100.

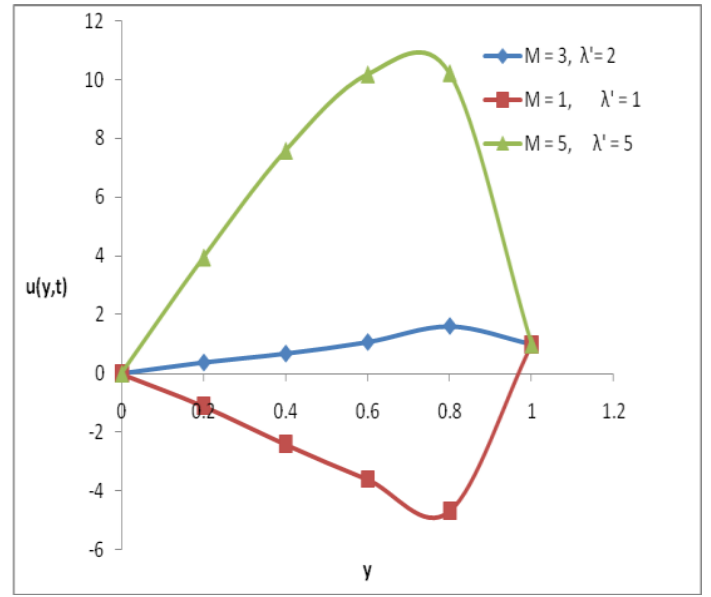

Fig. 3. Dimensionless velocity profiles against y at $\operatorname{Pr}=1000$ with different $M$ and $\lambda^{\prime}$.

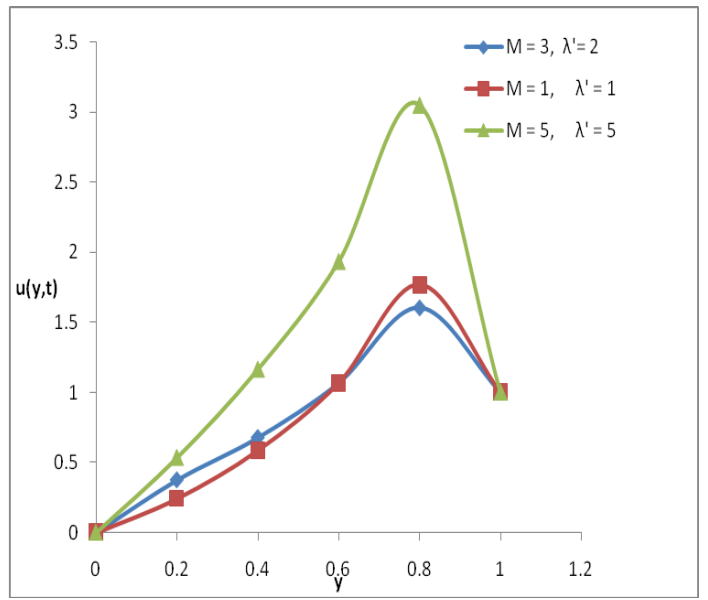

Fig. 4. Dimensionless velocity profiles against y at $\operatorname{Pr}=1000$ with different $\mathrm{M}$ and $\lambda^{\prime}$.

Figure 3 shows that velocity profiles increase with increase in Prandtl number when we have moderate permeability than the case of high permeability which is associated with very low velocity profile. The increase in Prandtl number translates to an increase in the viscosity of the conducting fluid. Viscous dissipation effect is more noticeable in this case as it increases the temperature of the fluid flow, coupled with radiative heat flux and this accounts for the very high temperature experienced by the fluid flow. This high temperature weakens the magnetic field strength which emanated from the magnetic field to increase velocity profile as the fluid flow is no more resisted by the magnetic field to such an extent that the conducting fluid flow loses its magnetic properties. Thereby, as Hartmann number increases the velocity profile increases when there is low and medium permeabilities in the transient state. It is worth mentioning that at high permeability of the medium, the velocity profile is very low.

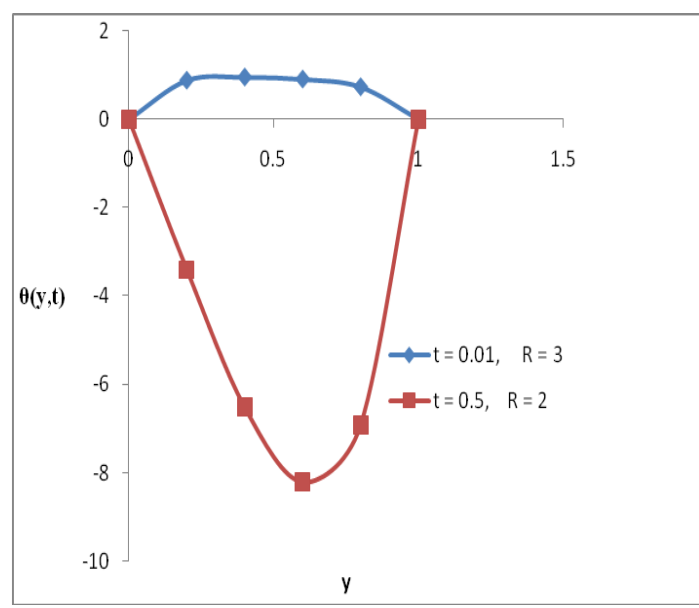

Fig. 5. Dimensionless temperature profiles against y at $\mathrm{Na}=100$.

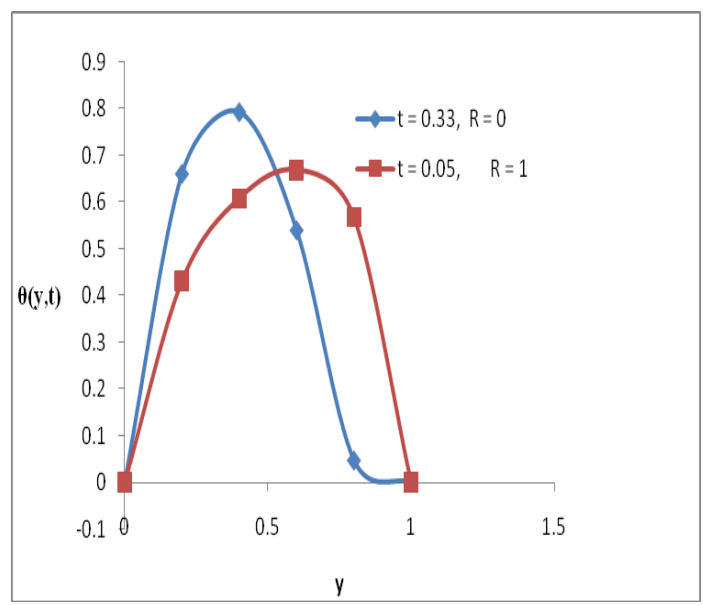

Fig. 6. Dimensionless temperature profiles against y at $\mathrm{Na}=10$.

Similarly, in Fig. 4, the similar effect is noticed when a high magnetic field of $\mathrm{M}=5$ is examined for moderate permeability while $\operatorname{Pr}=1000$ at $\mathrm{t}=0.0001$. The interaction of the radiative heat flux and magnetic field results in depolarization of the magnetic flux. These lines of forces with which the MHD fluid is frozen are broken and the fluid can no longer carry along the magnetic flux. The fluid also loses its conducting properties. At a point in short time step, when $\mathrm{M}=2$ with $\lambda^{\prime}=2$ and $M=1$ with $\lambda^{\prime}=1$, the velocity profiles in these cases are equal. Until the latter's velocity profile continues to lead that of the former. High porosity accounts for this phenomenon. Therefore, application of external magnetic field, at this time, reduces the velocity profile of the fluid flow. This is because of the fact that the magnetic field strength is unable to resist the flow as in the cases of Fig. 1 and Fig. 2 above. Therefore, at moderate permeability, higher Hartmann number shows considerable high 
velocity profiles. Figure 5 shows that there is corresponding increase in the temperature profiles as the thermal radiation increases in the fluid flow. This is because the thermal radiation is associated with high temperature, thereby increasing the temperature distribution of the fluid flow. When $\mathrm{R}=0$ at a time space of 0.33 , the results corresponds to that of Daskalaskis (1990) who carried out similar work without the presence of thermal radiation. Figure 6 further shows that increase in thermal radiation results to increase in temperature profile as in the case of Fig. 5. At some high time space, the temperature profiles continue to overshoot the boundary. Figures 5 and 6 also show that increase in Nahme number leads to increase in temperature profiles.

\section{Conclusions}

A numerical study has been carried out to investigate the influence of thermal radiation on transient MHD Couette flow of a high Prandtl fluid with temperaturedependent viscosity and thermal conductivity through a porous medium. The dimensionless governing equations are solved by an implicit finite difference method of Crank-Nicolson type. The following conclusions are made:

(i) The velocity profiles of the flow increase when the parameter Pr is increased.

(ii) Increase in thermal radiation of the fluid leads to increase in the temperature profiles.

(iii) Increase in permeability parameters results to an increase in temperature of the fluid but it reduces when the velocity of the fluid increases.

(iv) The temperature profiles of the flow increase as Nahme number increases.

(v) The velocity profiles for the flow maintain the boundary conditions of flow at very low time space but overshoot the boundary at moderate and high time steps.

(vi) Increasing Hartmann number increases the velocity profiles of the flow when $\operatorname{Pr}$ is high and decreases the velocity profiles $\mathrm{Pr}$ is low with significant effects of viscous heating, thermal radiation and permeability parameter.

(vii) The permeability of the porous medium and thermal radiation have insignificant effects on the transient MHD Couette fluid flow.

(viii) Finally, as stated in Daskalaskis (1990) for $\mathrm{t}=$ 0.33 , the presence of the porous medium provokes an overshoot of the temperature profile causing a considerable skewness of the distribution with a shift towards the mobile cylindrical surface, this corresponds with our result for $\mathrm{t}=0.33$ and $\mathrm{R}=0$

\section{REFERENCES}

Alagoa, K. D., G. Tay and T. M. Abbey (1999). Radiation and free convection effects on a MHD flow through a porous medium between infinite parallel plates with time- dependent suction. Astrophysics Space Science 260, 455 - 468.
Anjali Devi, S.P. and B. Ganga (2010). Dissipation effects on MHD nonlinear flow and heat transfer past a porous surface with prescribed heat flux. Journal of Applied Fluid Mechanics 3(1), 1 - 6.

Branover, H. (1978). Magnetohydrodynamic Flow in Ducts. John Wiley and Sons, New York.

Chamka, A. J. (2003). MHD flow of a uniformly stretched vertical permeable surface in the presence of heat generation/absorption and chemical reaction. International Communication Heat Mass Transfer 3, 413 - 422.

Chamka, A. J. (2000). Thermal radiation and buoyancy effects on hydromagnetic flow over an accelerating permeable surface with heat source or sink. International Journal of Engineering Sciences 38, $1699-1712$.

Cowling, T.G. (1957). Magnetohydrodynamics. Interscience, New York.

Daskalaski, J. (1990). Couette flow through a porous medium of a high Prandtl number fluid with temperature dependent viscosity. International Journal of Energy Research 14, 21-26.

Eckert, ERG and M. Faghri (1986). Viscous heating of high Prandtl number fluids with temperaturedependent viscosity. International Journal of Heat and Mass Transfer 29, 1177-1183.

Ganesan, P. and K. Ekambavanan (1992). Numerical solution of unsteady natural convection on isothermal inclined plate. Indian Journal of Technology 30, 471 - 476 .

Ganesan, P. and G. Palani (2002). Natural convection effects on an impulsively started isothermal inclined plate. Acta Mechanica 153, 127-132.

Gbadeyan, J.A. and M.S. Dada (1998). The effect of variable fluid properties and radiative MHD flow of a fluid in a vertical channel. Journal of Mathematical Association of Nigeria 26, 602-622.

Hazeem, A.A. (2006). Influence of temperature dependent viscosity on the MHD Couette flow of dusty fluid with heat transfer. Differential Equations and Non-linear Mechanics, Article ID 75290 .

Israel-Cookey, C. and F.B. Sigalo (2003). On the unsteady MHD free - convection flow past semiinfinite heated porous vertical Plate with timedependent suction and radiative heat transfer. AMSE Modeling, Simulating and Control B72, 2537.

Kreyzig, E. (2004). Advanced Engineering Mathematics, $8^{\text {th }}$ Edition, John Wiley and Sons, Inc., New York.

Makinde, O.D. and P.Y. Mhone (2005). Heat transfer to MHD oscillatory flow in a channel filled with 
I.G. Baoku et al. / JAFM, Vol. 5, No. 1, pp. 81-87, 2012.

porous medium. Romania Journal of Physics 50(9-10), 931-938.

Makinde, O.D. and E. Osalusi (2006). MHD steady flow in a channel with slip at the permeable boundaries. Romania Journal Physics 51 (3-4), $319-328$

Kim, M.C., K. Y. Kim and S. Kim (2004). The onset of transient convection in fluid saturated porous layer heated uniformly below. International Communication Heat Mass Transfer 31(1), 53-62.

Osalusi, E. and P. Sibanda (2006). On variable laminar convection flow properties due to a porous rotating disk in a magnetic field. Romania Journal of Physics 51(9-10), 937 - 950.

Raptis, A. and C.V. Massalas (1998). Magnetohydrodynamic flow past a plate by the presence of radiation. Heat and Mass Transfer 34, $107-109$.

Sharma, P. R. and P. Mathur (1995). Steady laminar free convection flow of an electrically conducting fluid along a porous hot vertical plate in the presence of heat source / sink. Indian Journal of Pure and Applied Mathematics 26 (11), 1125 1134.

Sharma, P.R. and G. Singh (2009). Effects of variable thermal conductivity and heat source/sink on MHD flow near a stagnation point on a linearly stretching sheet, Journal of Applied Fluid Mechanics 2(1), 13 - 21.

Takhar, H.S., A.J. Chamkha and G. Nath (2001). Unsteady laminar MHD flow and heat transfer in the stagnation region of an impulsively spinning and translating sphere in the presence of buoyancy forces. International Journal of Heat and Mass Transfer 37, 397-402.

Takhar, H.S., RSR Gorla, and V.M. Soundalgekar (1996). Radiative effects on MHD free convection flow of a gas past a semi-infinite vertical plate. International Journal of Numerical Heat Fluid Flow 2(2), 77-83. 